\title{
Une méthode semi-analytique pour l'analyse de l'interaction entre une onde de choc et une coque élastique immergée. Application au pré-dimensionnement de coques de sous-marins
}

\author{
Jean-François Sigrista ${ }^{a}$ et Cédric Leblond \\ Centre Propulsion de DCNS, Service Technique et Scientifique, 44620 La Montagne, France
}

Reçu le 27 février 2008, accepté le 11 décembre 2008

\begin{abstract}
Résumé - Le dimensionnement des structures immergées vis-à-vis des effets d'une explosion sous-marine constitue une exigence forte pour un constructeur de navires militaires. La présente étude s'intéresse à la description de l'interaction entre une coque élastique immergée et une onde acoustique se propageant dans le fluide. En utilisant un modèle linéaire pour le fluide et la structure, il est possible de proposer une description du problème couplé dont la résolution peut être conduite avec une méthode semi-analytique. Les inconnues du problème (pour le fluide : champ de pression incident, champ de pression réfléchi, champ de pression rayonné ; pour la structure : champ de déplacement radial et ortho radial) sont écrites sous la forme d'un développement en série de Fourier de la variable angulaire. L'évolution des différents champs est décrite dans le domaine de Laplace par transformation des équations temporelles du problème couplé. Une application est proposée ici au cas de deux coques couplées par un fluide. La méthode de calcul ainsi proposée est implantée dans un code de calcul MATLAB qui peut ainsi être utilisé en bureau d'études pour le pré-dimensionnement de coques de sous-marins.
\end{abstract}

Mots clés : Interaction fluide-structure / explosion sous-marine / onde de choc / coque immergée / méthode semi-analytique

\begin{abstract}
A semi-analytical approach for the modelling of acoustic shock wave-elastic submerged shell interaction. Application to the pre-design of submarine hulls. Numerical simulation of acoustic shock wave/elastic structure interaction is of paramount importance in naval shipbuilding. The present study deals with the numerical calculation of the fluid-structure interaction between a pressure wave and an elastic shell for a 2D case. The modelling accounts for incident reflected and scattered pressure fields in the fluid medium. In such conditions, an analytical formulation of the coupled problem can be derived using a Fourier serie to solve the spatial dependence and a Laplace transform to solve the time dependence of the displacement and pressure fields. The present approach provides a powerful and accurate method that accounts for interaction phenomena on simple cases. The method is applied on the case of two elastic shells coupled by a fluid and subjected to shock wave loading. The proposed calculation procedure is implemented in a MATLAB code which can be used in pre-design stages to investigate the dynamic behaviour of submerge shells under shock wave loading.
\end{abstract}

Key words: Fluid-structure interaction / underwater explosion / shock wave / submerged shell / semi-analytical method

\section{Introduction}

Le dimensionnement des navires militaires requiert l'analyse du comportement d'ensemble aux effets d'une explosion sous-marine non au contact se produisant à proximité du navire (choc hydrodynamique, voir Fig. 1)

\footnotetext{
a Auteur pour correspondance :

jean-francois.sigrist@dcnsgroup.fr
}

et constitue ainsi un des défis techniques de première importance en construction navale militaire. Le choc hydrodynamique et ses effets sur des structures immergées mettent en jeu un ensemble de phénomènes couplés fluide/structure dont la modélisation mathématique et l'analyse physique par simulations numériques ou essais empiriques font l'objet d'études importantes et constituent à ce jour un problème dont l'analyse complète n'est toujours pas à portée de l'ingénieur. 


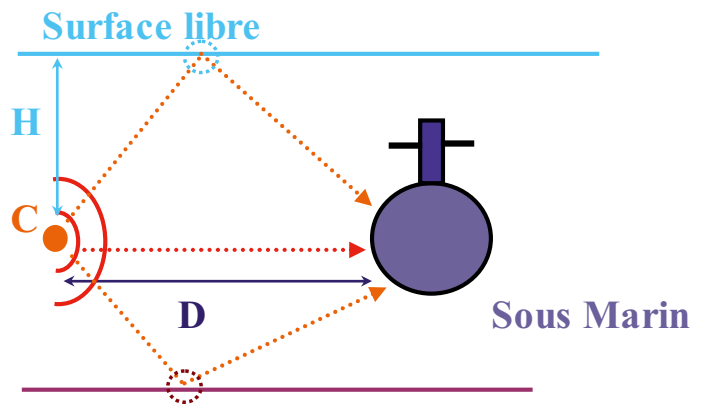

Fond

Fig. 1. Choc hydrodynamique sur un sous-marin : explosion d'une charge $C$ à une distance $D$ du bordée du navire. Effets de l'explosion : chargements direct ou indirect en pression dus à la présence de la surface libre ou des fonds marins.

La compréhension de la physique en jeu et le développement de méthodes numériques permettant sa simulation ont pour but de rendre compte du chargement subi par le navire et de définir des données d'entrées pour le dimensionnement des structures navales (coques et équipements).

La caractérisation de l'explosion sous-marine [1] et de ses effets sur des structures immergées simples est maintenant connue avec une précision acceptable pour l'ingénieur, en particulier grâce à un grand nombre d'études analytiques ou numériques réalisées au cours des dernières décennies $[2-8]$.

La description complète du phénomène [9], de l'explosion de la charge sous-marine jusqu'à la réponse d'un équipement embarqué, nécessite en revanche la description de la détonation (modélisation chimique et thermodynamique), de la propagation de l'onde de choc primaire et secondaire qui en résulte (modélisation en dynamique des fluides), des effets d'interaction fluide/structure au moment de l'impact de l'onde de choc sur la coque (modélisation dynamique couplée du fluide et de la structure) et de la propagation de l'onde de choc dans les structures telles que berceau, plots de suspension et équipements (modélisation en dynamique linéaire et nonlinéaire des structures). Les phénomènes dynamiques interviennent à des échelles de temps et d'espace très étendues (de quelques millisecondes pour l'explosion à quelques secondes pour la réponse du navire, de quelques dizaines de mètres pour le navire à quelques dizaines de centimètres pour un équipement).

La variété des manifestations physiques mises en jeu dans un tel problème ne peut être appréhendée dans son intégralité que par un essai sur le navire, voir figure 2 l'exemple de l'essai de choc réalisé par DCNS sur le premier navire de la série des frégates type « La Fayette». L'analyse des résultats de l'essai à échelle réelle [10] permet de disposer de données en terme de chargement sur coque et de réponse des équipements embarqués; ces données peuvent alors servir aussi bien à la spécification qu'à la qualification des structures.
Le recours à un essai à échelle réelle (ou échelle réduite) sur le navire (ou sur un équipement) n'est cependant pas envisageable de façon systématique, en raison des contraintes techniques et financières associées à la préparation de l'essai. Les possibilités offertes par la simulation numérique permettent ainsi d'envisager l'analyse des phénomènes dynamiques en jeu lors du choc hydrodynamique au moyen de codes de calculs (ou 《hydrocodes ») [11-13], dont le développement résulte d'un important travail de modélisation mathématique et numérique [14-17], rendus accessibles à l'ingénieur pour des applications à des projets industriels, sur des modèles de plus en plus détaillés de navires [18-22]. La simulation numérique s'impose alors de plus en plus dans l'ingénierie comme un moyen d'analyse a priori (en phase de dimensionnement) ou a posteriori (en phase d'exploitation) des structures navales [23], voir figure 2 l'exemple de la simulation numérique de l'essai de choc de la frégate « La Fayette $»$.

La mise en œuvre de calculs numériques requiert cependant une connaissance précise de la géométrie du navire et nécessite des temps de modélisation et de calcul très importants. En phase d'avant projet, il est pertinent d'utiliser des modélisations simplifiées de l'interaction onde de choc/structure élastique. Ce point a ainsi fait l'objet de nombreuses études depuis les premiers travaux engagés sur le sujet ${ }^{1}$. La présente étude s'inscrit ainsi dans cette démarche et a pour objet de développer des modèles simples utilisables en bureau d'étude pour une définition des données d'entrée en phase de pré-dimensionnement. Dans une approche globale, le choc est défini par le facteur de choc $K=\sqrt{C} / D$, où $C$ est la charge de l'explosif et $D$ la distance au navire [1], voir figure 1 . Il s'agit alors de calculer la réponse d'une structure simple soumise à cette onde de pression pour un facteur de choc donné.

\section{Modélisation mathématique et résolution analytique}

Le problème générique étudié est défini par la figure 3 : on s'intéresse au cas de deux coques élastiques circulaires, couplées par un fluide contenu dans l'espace annulaire formé par les deux coques et baignant dans un fluide externe supposé infini. La charge explosive de masse $C$ est positionnée à la distance $D$ de la coque extérieure. Ce modèle est une schématisation d'une tranche représentant l'ensemble coque résistante/coque hydrodynamique d'un sous-marin : la modélisation proposée reprend les hypothèses formulées dans les travaux de Iakovlev, qui a étudié l'interaction entre des coques de révolution et une onde de choc sphérique $[24]^{2}$. On restreint ici l'étude à

\footnotetext{
1 Parmi ces études dont certaines références ont été citées ci-avant, on peut indiquer les travaux récents de Iakovlev, voir par exemple [24,25].

2 À la connaissance des auteurs, le cas considéré ici (deux coques immergées couplées par un fluide et soumises à une onde de pression) n'a pas à ce jour fait l'objet d'études particulières.
} 


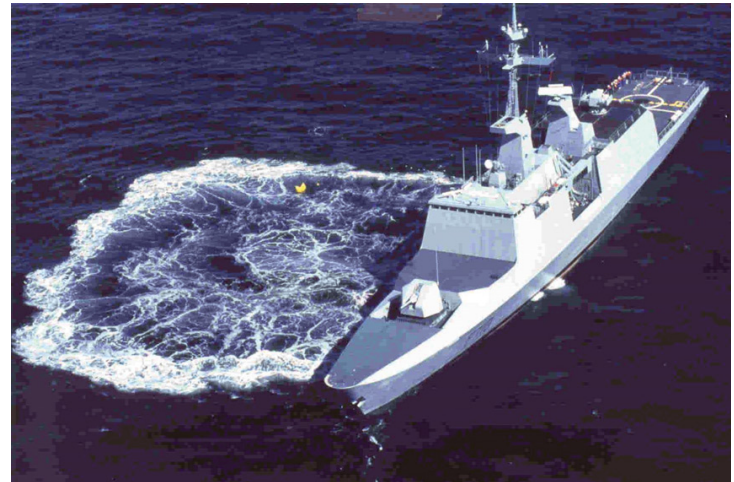

De l'essai de choc réel...

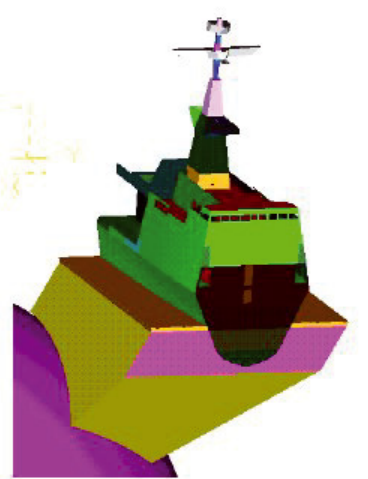

...à sa simulation numérique

Fig. 2. Essai de choc réel sur la frégate de type « La Fayette » et simulation numérique de l'essai.

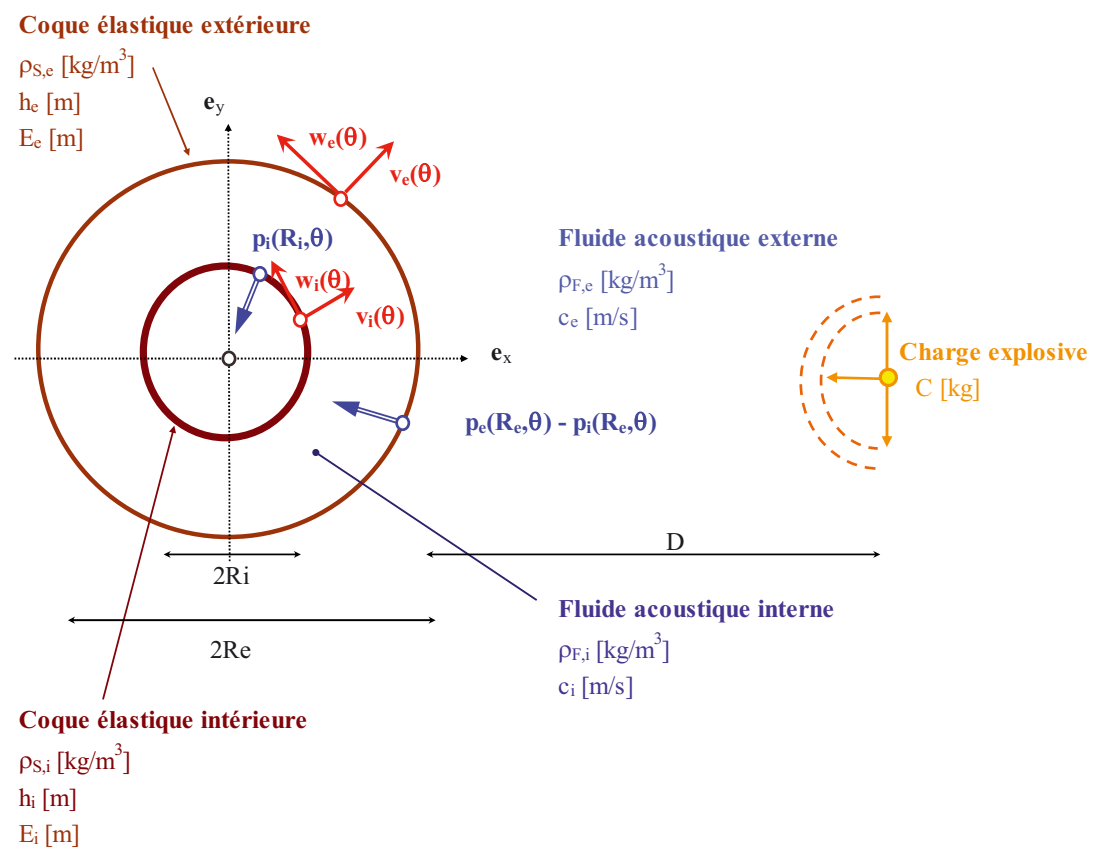

Fig. 3. Deux coques élastiques couplées par un fluide (intérieur), immergées dans un fluide (extérieur) et soumises à une onde de pression résultant de l'explosion d'une charge $C$ à distance $D$.

une géométrie bidimensionnelle et une approche linéaire du comportement de la structure et du fluide.

En particulier, les effets de pulsation de bulle et de jet au voisinage des parois [26], de dissipation visqueuse dans le fluide et de décollement de l'écoulement autour de la paroi [27], de cavitation dans le fluide [28] et de déformations/contraintes tridimensionnelles [25] ne sont pas pris en compte dans la modélisation; l'ensemble de ces points fait l'objet d'études en cours à DCNS.

La modélisation reste donc assez frustre mais répond à l'objectif industriel initial de disposer de modèles d'interaction fluide/structure élémentaires, pour laquelle une résolution exacte ${ }^{3}$ est mise en œuvre.

\footnotetext{
${ }^{3}$ La résolution proposée est qualifiée d'exacte au sens où elle n'utilise pas d'approximations asymptotiques type «DAA $»$ ou « Early-time » $[16,17]$.
}

\subsection{Modélisation mathématique}

Le comportement du système couplé est décrit par les équations d'un anneau élastique bidimensionnel et d'un fluide acoustique [29]. On utilise les notations de la figure 3 , soit pour les structures : $\rho_{S}$ (masse volumique), $E$ (module d'Young), $R$ (rayon) et $h$ (épaisseur) ${ }^{4}$, pour les fluides : $\rho_{\mathrm{F}}$ (masse volumique) et $c$ (célérité du son); les composantes radiale et ortho-radiale du déplacement de la structure sont notées respectivement $\nu(\theta, t)$ et $w(\theta, t)$, le champ de pression dans le fluide est noté $p(r, \theta, t)$. Les indices $i$ et $e$ désignent respectivement

\footnotetext{
${ }^{4}$ On se place dans l'hypothèse de coques minces $(h / R \ll 1)$, ce qui n'est pas forcément vérifié dans le cas réel. Une extension de la modélisation présentée ici est également à l'étude pour prendre en compte le comportement de coques épaisses [30].
} 
les structures/fluides intérieurs et extérieurs. Avec les hypothèses de comportement élastique des structures et acoustique des fluides, les équations du système couplé sont les suivantes.

\section{Problème structure}

- Coque extérieure

$$
\begin{aligned}
\rho_{S \mathrm{e}} h_{\mathrm{e}} \frac{\partial^{2} \nu_{\mathrm{e}}}{\partial t^{2}}+\frac{E_{\mathrm{e}} h_{\mathrm{e}}}{R_{\mathrm{e}}^{2}}\left[\nu_{\mathrm{e}}+\frac{\partial w_{\mathrm{e}}}{\partial \theta}\right. & \left.+\frac{h_{\mathrm{e}}^{2}}{12 R_{\mathrm{e}}^{2}}\left(\frac{\partial^{4} \nu_{\mathrm{e}}}{\partial \theta^{4}}-\frac{\partial^{3} w_{\mathrm{e}}}{\partial \theta^{3}}\right)\right] \\
& =-\left.p_{\mathrm{e}}\right|_{r=R_{\mathrm{e}}}+\left.p_{\mathrm{i}}\right|_{r=R_{\mathrm{e}}}
\end{aligned}
$$

$$
\begin{aligned}
\rho_{S \mathrm{e}} h_{\mathrm{e}} \frac{\partial^{2} w_{\mathrm{e}}}{\partial t^{2}}-\frac{E_{\mathrm{e}} h_{\mathrm{e}}}{R_{\mathrm{e}}^{2}}[ & \frac{\partial^{2} w_{\mathrm{e}}}{\partial \theta^{2}}+\frac{\partial \nu_{\mathrm{e}}}{\partial \theta} \\
& \left.+\frac{h_{\mathrm{e}}^{2}}{12 R_{\mathrm{e}}^{2}}\left(\frac{\partial^{2} w_{\mathrm{e}}}{\partial \theta^{2}}-\frac{\partial^{3} \nu_{\mathrm{e}}}{\partial \theta^{3}}\right)\right]=0
\end{aligned}
$$

- Coque intérieure

$$
\begin{aligned}
\rho_{S \mathrm{i}} h_{\mathrm{i}} \frac{\partial^{2} \nu_{\mathrm{i}}}{\partial t^{2}}+\frac{E_{\mathrm{i}} h_{\mathrm{i}}}{R_{\mathrm{i}}^{2}} & {\left[\nu_{\mathrm{i}}+\frac{\partial w_{\mathrm{i}}}{\partial \theta}\right.} \\
& \left.+\frac{h_{\mathrm{i}}^{2}}{12 R_{\mathrm{i}}^{2}}\left(\frac{\partial^{4} \nu_{\mathrm{i}}}{\partial \theta^{4}}-\frac{\partial^{3} w_{\mathrm{i}}}{\partial \theta^{3}}\right)\right]=-\left.p_{\mathrm{i}}\right|_{r=R_{\mathrm{i}}}
\end{aligned}
$$

$$
\begin{aligned}
\rho_{S \mathrm{i}} h_{\mathrm{i}} \frac{\partial^{2} w_{\mathrm{i}}}{\partial t^{2}}-\frac{E_{\mathrm{i}} h_{\mathrm{i}}}{R_{\mathrm{i}}^{2}}\left[\frac{\partial^{2} w_{\mathrm{i}}}{\partial \theta^{2}}+\frac{\partial \nu_{\mathrm{i}}}{\partial \theta}\right. & \\
& \left.+\frac{h_{\mathrm{i}}^{2}}{12 R_{\mathrm{i}}^{2}}\left(\frac{\partial^{2} w_{\mathrm{i}}}{\partial \theta^{2}}-\frac{\partial^{3} \nu_{\mathrm{i}}}{\partial \theta^{3}}\right)\right]=0
\end{aligned}
$$

\section{Problème fluide}

- Fluide extérieur

$$
\begin{gathered}
\frac{1}{c_{\mathrm{e}}^{2}} \frac{\partial^{2} p_{\mathrm{e}}}{\partial t^{2}}-\Delta p_{\mathrm{e}}=0 \\
\left.\frac{\partial p_{\mathrm{e}}}{\partial r}\right|_{r=R_{\mathrm{e}}}=-\rho_{\mathrm{F}}^{\mathrm{e}} \frac{\partial^{2} \nu_{\mathrm{e}}}{\partial t^{2}},\left.\quad p_{\mathrm{e}}\right|_{r \rightarrow \infty}=0
\end{gathered}
$$

- Fluide intérieur

$$
\begin{gathered}
\frac{1}{c_{\mathrm{i}}^{2}} \frac{\partial^{2} p_{\mathrm{i}}}{\partial t^{2}}-\Delta p_{\mathrm{i}}=0 \\
\left.\frac{\partial p_{\mathrm{i}}}{\partial r}\right|_{r=R_{\mathrm{e}}-h_{\mathrm{e}}}=-\rho_{\mathrm{Fi}} \frac{\partial^{2} \nu_{\mathrm{e}}}{\partial t^{2}},\left.\quad \frac{\partial p_{\mathrm{i}}}{\partial r}\right|_{r=R_{\mathrm{i}}}=-\rho_{\mathrm{Fi}} \frac{\partial^{2} \nu_{\mathrm{i}}}{\partial t^{2}}
\end{gathered}
$$

Les équations (6) et (8) donnent les conditions de couplage des coques avec les fluides, ainsi que la condition de décroissance de la pression à l'infini.

\subsection{Résolution semi-analytique}

La méthode de résolution numérique du problème posé par les équations (1)-(8) suit une démarche classique et s'effectue en trois temps :

i) les équations couplées (1) à (8) sont écrites sous forme adimensionnelle, en utilisant comme longueur caractéristique le rayon de la coque extérieure $L \equiv R_{\mathrm{e}}$, comme vitesse caractéristique la célérité du son dans la coque extérieure $L T^{-1} \equiv \sqrt{E_{\mathrm{e}} / \rho_{S \mathrm{e}}}$ et comme contrainte caractéristique le module de compressibilité du fluide extérieur $M L^{-1} T^{-2} \equiv \rho_{F \mathrm{e}} c_{\mathrm{e}}^{2}$. Les champs de déplacement et de pression adimensionnels sont ainsi notés $\nu_{\mathrm{e} / \mathrm{i}}^{*}\left(t^{*}\right), w_{\mathrm{e} / \mathrm{i}}^{*}\left(t^{*}\right)$ et de pression $p_{\mathrm{e} / \mathrm{i}}^{*}\left(t^{*}\right)$;

ii) les équations adimensionnelles sont transformées dans le domaine de Laplace. Les transformées de Laplace des champs adimensionnels sont notés $V_{\mathrm{e} / \mathrm{i}}^{*}\left(s^{*}\right)$, $W_{\mathrm{e} / \mathrm{i}}^{*}\left(t^{*}\right)$ et de $P_{\mathrm{e} / \mathrm{i}}^{*}\left(s^{*}\right)$;

iii) les équations transformées sont projetées sur les composantes de Fourier symétriques et antisymétriques suivant la variable angulaire $\theta$. Les composantes de Fourier d'ordre $n$ des transformées de Laplace adimensionnelles sont notées $V_{n, \mathrm{e} / \mathrm{i}}^{*}\left(s^{*}\right), W_{n, \mathrm{e} / \mathrm{i}}^{*}\left(t^{*}\right)$ et de $P_{n, \mathrm{e} / \mathrm{i}}^{*}\left(s^{*}\right)$.

En introduisant les nombres sans dimension suivants : $M_{\mathrm{e} / \mathrm{i}}=\rho_{\mathrm{Fe} / \mathrm{i}} / \rho_{\mathrm{Se} / \mathrm{i}}, C_{\mathrm{e} / \mathrm{i}}=c_{\mathrm{e} / \mathrm{i}} \sqrt{\rho_{\mathrm{Se} / \mathrm{i}} / E_{\mathrm{e} / \mathrm{i}}}, E_{\mathrm{e} / \mathrm{i}}=$ $h_{\mathrm{e} / \mathrm{i}} / R_{\mathrm{e} / \mathrm{i}}, L_{\mathrm{e} / \mathrm{i}}=h_{\mathrm{e} / \mathrm{i}}^{2} / 12 R_{\mathrm{e} / \mathrm{i}}^{2}, \mu=\rho_{\mathrm{Fe}} / \rho_{\mathrm{Fi}}, \lambda=R_{\mathrm{e}} / R_{\mathrm{i}}$, $\omega=c_{\mathrm{e}} / c_{\mathrm{i}}$, on montre alors que les champs de pression dans le fluide intérieur et extérieur s'expriment respectivement comme [30] :

$$
\begin{aligned}
P_{n, \mathrm{i}}^{*}\left(r^{*}, s^{*}\right)= & \frac{1}{C_{\mathrm{i}}^{2}} \frac{\omega^{2}}{\mu \gamma^{2}}\left(\Pi_{n, \mathrm{e}}^{*}\left(r^{*}, s^{*}\right) s^{* 2} V_{n, \mathrm{e}}^{*}\right. \\
& \left.+\Pi_{n, \mathrm{i}}^{*}\left(r^{*}, s^{*}\right) s^{* 2} V_{n, \mathrm{i}}^{*}\right) \\
P_{n, \mathrm{e}}^{*}\left(r^{*}, s^{*}\right)= & \left.P_{n, \mathrm{o}}^{*}\right|_{r^{*}=1} \\
& +\Pi_{n, \mathrm{o}}^{*}\left(r^{*}, s^{*}\right)\left(\frac{1}{C_{\mathrm{e}}^{2}} s^{* 2} V_{n, \mathrm{e}}^{*}+\left.\frac{\partial P_{n, \mathrm{o}}^{*}}{\partial r^{*}}\right|_{r^{*}=1}\right)
\end{aligned}
$$

avec :

$$
\Pi_{n, \mathrm{o}}^{*}\left(r^{*}, s^{*}\right)=-\frac{K_{n}\left(s^{*} / r^{*} C_{\mathrm{e}}\right)}{s^{*} / C_{\mathrm{e}} K_{n}^{\prime}\left(s^{*} r^{*} / C_{\mathrm{e}}\right)}
$$

$\Pi_{n, \mathrm{e}}^{*}\left(r^{*}, s^{*}\right)=\frac{1}{\omega s^{*} / C_{\mathrm{i}}}$

$\times \frac{K_{n}^{\prime}\left(\omega s^{*} \lambda / C_{\mathrm{i}}\right) I_{n}\left(\omega s^{*} r^{*} / C_{\mathrm{i}}\right)-I_{n}^{\prime}\left(\omega s^{*} / \lambda C_{\mathrm{i}}\right) K_{n}\left(\omega s^{*} r^{*} / C_{\mathrm{i}}\right)}{K_{n}^{\prime}\left(\omega s^{*} / \lambda C_{\mathrm{i}}\right) I_{n}^{\prime}\left(\omega s^{*} / C_{\mathrm{i}}\right)-I_{n}^{\prime}\left(\omega s^{*} / \lambda C_{\mathrm{i}}\right) K_{n}^{\prime}\left(\omega s^{*} / C_{\mathrm{i}}\right)}$

$\Pi_{n, \mathrm{i}}^{*}\left(r^{*}, s^{*}\right)=\frac{1}{\omega s^{*} / C_{\mathrm{i}}}$

$\times \frac{I_{n}^{\prime}\left(\omega s^{*} / C_{\mathrm{i}}\right) K_{n}\left(\omega s^{*} r^{*} / C_{\mathrm{i}}\right)-K_{n}^{\prime}\left(\omega s^{*} / C_{\mathrm{i}}\right) I_{n}\left(\omega s^{*} r^{*} / C_{\mathrm{i}}\right)}{K_{n}^{\prime}\left(\omega s^{*} / \lambda C_{\mathrm{i}}\right) I_{n}^{\prime}\left(\omega s^{*} / C_{\mathrm{i}}\right)-I_{n}^{\prime}\left(\omega s^{*} / \lambda C_{\mathrm{i}}\right) K_{n}^{\prime}\left(\omega s^{*} / C_{\mathrm{i}}\right)}$ 


$$
\begin{aligned}
& s^{* 2}\left[\begin{array}{cccc}
1+\frac{M_{\mathrm{e}}}{E_{\mathrm{e}}}\left(\Pi_{n, \mathrm{o}}^{*}\left(1, s^{*}\right)+\Pi_{n, \mathrm{e}}^{*}\left(1, s^{*}\right) / \mu\right) & 0 & \frac{M_{\mathrm{e}}}{E_{\mathrm{e}}} \Pi_{n, \mathrm{i}}^{*}\left(1, s^{*}\right) / \mu & 0 \\
0 & 1 & 0 & 0 \\
\frac{\omega^{2}}{\lambda^{2}} \frac{M_{\mathrm{i}}}{E_{\mathrm{i}}} \lambda \Pi_{n, \mathrm{e}}^{*}\left(1 / \lambda, s^{*}\right) & 0 \frac{\omega^{2}}{\lambda^{2}}\left(1+\frac{M_{\mathrm{i}}}{E_{\mathrm{i}}} \lambda \Pi_{n, \mathrm{i}}^{*}\left(1 / \lambda, s^{*}\right)\right) & 0 \\
0 & 0 & 0 & \frac{\omega^{2}}{\lambda^{2}}
\end{array}\right]\left\{\begin{array}{c}
V_{n, \mathrm{e}}^{*} \\
W_{n, \mathrm{e}}^{*} \\
V_{n, \mathrm{i}}^{*} \\
W_{n, \mathrm{i}}^{*}
\end{array}\right\} \\
& +\left[\begin{array}{cccc}
1+n^{4} L_{\mathrm{e}} & n\left(1+n^{2} L_{\mathrm{e}}\right) & 0 & 0 \\
n\left(1+n^{2} L_{\mathrm{e}}\right) & n^{2}\left(1+L_{\mathrm{e}}\right) & 0 & 0 \\
0 & 0 & 1+n^{4} L_{\mathrm{i}} & n\left(1+n^{2} L_{\mathrm{i}}\right) \\
0 & 0 & n\left(1+n^{2} L_{\mathrm{i}}\right) & n^{2}\left(1+L_{\mathrm{i}}\right)
\end{array}\right]\left\{\begin{array}{c}
V_{n, \mathrm{e}}^{*} \\
W_{n, \mathrm{e}}^{*} \\
V_{n, \mathrm{i}}^{*} \\
W_{n, \mathrm{i}}^{*}
\end{array}\right\}=\left\{\begin{array}{c}
-\frac{M_{\mathrm{e}} C_{\mathrm{e}}^{2}}{E_{\mathrm{e}}}\left(\left.P_{n, \mathrm{o}}^{*}\right|_{r^{*}=1}+\left.\Pi_{n, \mathrm{o}}^{*}\left(1, s^{*}\right) \frac{\partial P_{n, \mathrm{o}}^{*}}{\partial r^{*}}\right|_{r *=1}\right) \\
0 \\
0 \\
0
\end{array}\right\}
\end{aligned}
$$

En reportant la valeur du champ de pression au niveau des parois des coques extérieure $\left(\right.$ en $\left.r^{*}=1\right)$ et intérieure $\left(\right.$ en $\left.r^{*}=1 / \lambda\right)$ on établit les systèmes d'équations couplées suivantes décrivant la dynamique des coques :

$$
\text { voir équation (14) ci-dessus }
$$

Les nombres sans dimension caractérisant la physique de l'interaction (Éqs. (9) à (14)) sont définis à partir des ratios $M_{\mathrm{e} / \mathrm{i}}, C_{\mathrm{e} / \mathrm{i}}, E_{\mathrm{e} / \mathrm{i}}=h_{\mathrm{e} / \mathrm{i}} / R_{\mathrm{e} / \mathrm{i}}, L_{\mathrm{e} / \mathrm{i}}, \mu, \lambda, \omega$ explicites précédemment.

L'algorithme de résolution du problème couplé est alors le suivant :

i) $V_{n, \mathrm{e} / \mathrm{i}}^{*}\left(s^{*}\right)$ et $W_{n, \mathrm{e} / \mathrm{i}}^{*}\left(s^{*}\right)$ sont calculés par inversion matricielle du système (14);

ii) $P_{n, \mathrm{e} / \mathrm{i}}^{*}\left(s^{*}\right)$ est calculée à l'aide des relations $(9)$ et $(10)$;

iii) l'inversion de Laplace permet de calculer les composantes de Fourier des champs de déplacement $v_{n, \mathrm{e} / \mathrm{i}}^{*}\left(t^{*}\right), w_{n, \mathrm{e} / \mathrm{i}}^{*}\left(t^{*}\right)$ et de pression $p_{n, \mathrm{e} / \mathrm{i}}^{*}\left(t^{*}\right)$. Cette opération est réalisée numériquement à l'aide d'algorithmes spécifiques développés dans MATLAB [31];

iv) les champs dimensionnels se déduisent à partir de la recombinaison de $N$ modes de Fourier des champs adimensionnels.

L'ensemble de la procédure est implémenté dans MATLAB sous forme d'un code de calcul dédié, qui peut être utilisé pour le calcul de la réponse d'une coque seule ou de deux coques couplées, soumise au champ de pression primaire de l'explosion sous-marine (voir Fig. 4). Le code de calcul permet en particulier le post-traitement et la visualisation des champs de pression dans les fluides et des déformations des coques.

Le chargement de la structure est défini par la donnée de la pression incidente $p_{\mathrm{o}}$ et du gradient de pression $\frac{\partial p_{\mathrm{o}}}{\partial r}$ incident à la paroi en $r=R_{\mathrm{e}}$, équation (14). La modélisation adoptée ici est similaire à celle de
Iakovlev [25] : sur l'axe $\theta=0$ le champ de pression est donné par l'expression :

$$
\begin{array}{r}
p_{o}(r, \theta=0, t)=\Pi \exp \left(-\frac{t-\left(r-R_{\mathrm{e}}\right) / c_{\mathrm{e}}}{\tau}\right) \\
H\left(\frac{t-\left(r-R_{\mathrm{e}}\right) / c_{\mathrm{e}}}{\tau}\right)
\end{array}
$$

où $H(\bullet)$ est la fonction de Heaviside; on suppose l'origine des temps $t=0$ comme étant l'instant où l'onde de pression impacte la coque extérieure. Le champ de pression au point d'impact est donc régi par une exponentielle décroissante $p\left(r=R_{\mathrm{e}}, \theta=0, t\right)=\Pi \exp (-t / \tau)$ où la pression maximum $\Pi$ et le temps de décroissance $\tau$ dépendent des paramètres $C, D$ de l'explosion selon [1] :

$$
\Pi=\Pi_{\mathrm{o}}\left[\frac{C^{a}}{D}\right]^{b} \quad \tau=\tau_{\mathrm{o}} C^{a}\left[\frac{C^{a}}{D}\right]^{c}
$$

où $\Pi_{\mathrm{o}}, \tau_{\mathrm{o}}, a, b$ et $c$ sont des paramètres dépendant de l'explosif. L'expression du gradient de pression est ensuite déduite de (15), et les composantes de Fourier sont calculées par définition selon

$p_{\mathrm{o}, n}(r=R, t)=\int_{0}^{2 \pi} p_{\mathrm{o}}(r=R, \theta, t) \cos (n \theta) \mathrm{d} \theta / \int_{0}^{2 \pi} \cos ^{2}(n \theta) \mathrm{d} \theta$.

La dépendance en $\theta$ est déterminée par une loi de propagation linéaire de l'onde sphérique en $1 / r$ à partir du point d'impact au droit de la coque extérieure.

Sur le plan numérique, il faut noter que la méthode de calcul proposée, qui est basée sur l'utilisation de la transformée de Laplace et son inversion numérique s'avère particulièrement efficace pour traiter les cas proposés. Sur des cas similaires, Iakovlev $[24,25]$ utilise une intégration analytique en temps des équations obtenues, qui trouve 


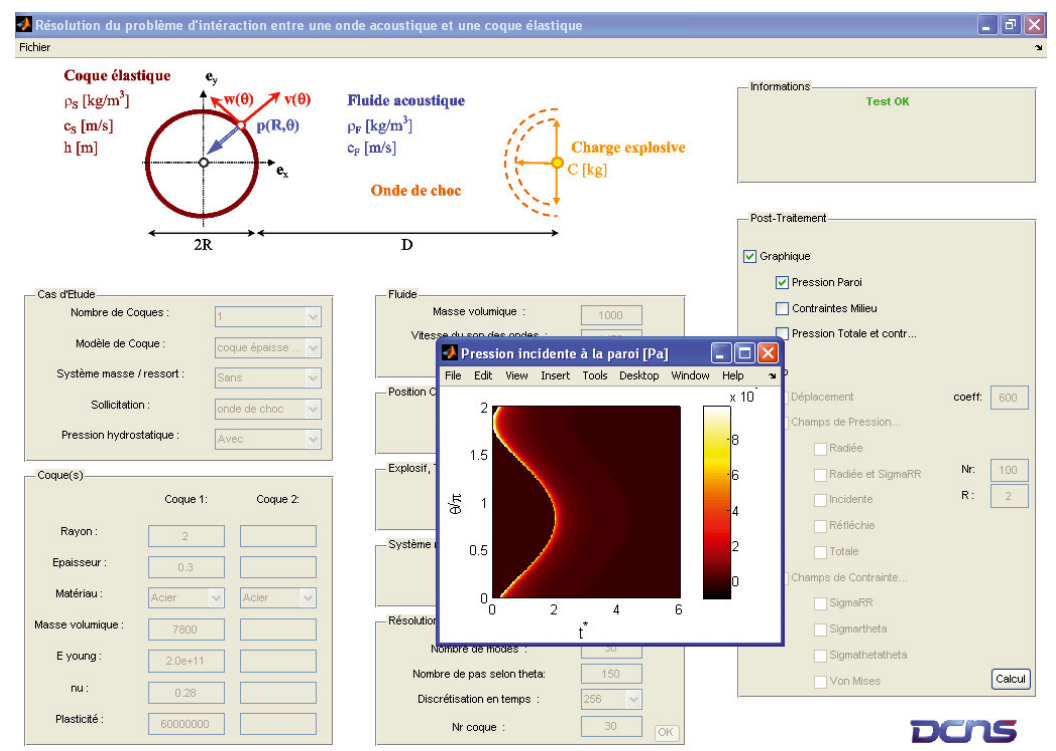

Fig. 4. Interface du code DCNS de calcul de l'interaction.

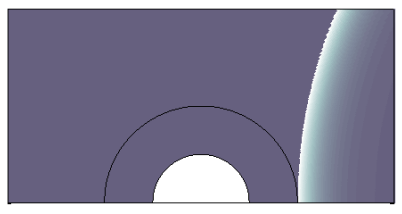

(a) $\mathrm{t}=0,00 \mathrm{~ms}$

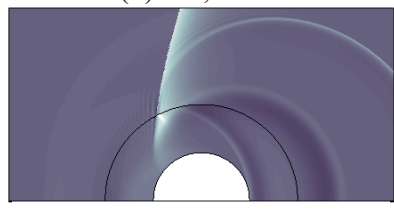

(e) $\mathrm{t}=1,41 \mathrm{~ms}$

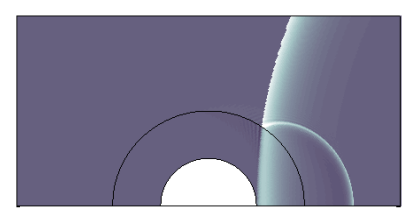

(b) $\mathrm{t}=0,35 \mathrm{~ms}$

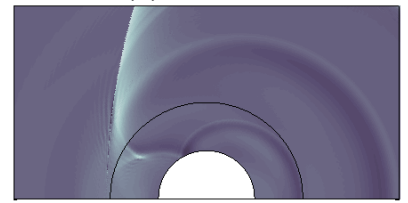

(d) $\mathrm{t}=1,06 \mathrm{~ms}$

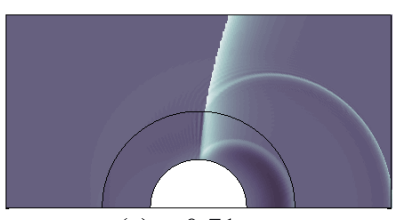

(c) $\mathrm{t}=0,71 \mathrm{~ms}$

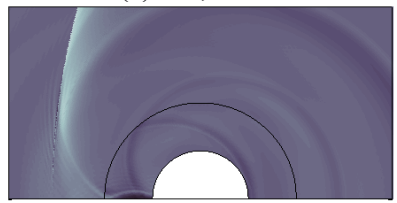

(f) $\mathrm{t}=1,76 \mathrm{~ms}$

Fig. 5. Simulation numérique de l'interaction onde de choc/coque élastique dans le cas de deux coques couplées par un fluide acoustique. Pression dans le fluide à différents instants.

rapidement ses limites en raison de problèmes de stabilité/précision numérique, en particulier dans le calcul des fonctions de type $K_{n}(z) / z K_{n}^{\prime}(z), I_{n}(z) / z I_{n}^{\prime}(z)$ ou $1 / z\left(K_{n}^{\prime}(z) I_{n}(z)-K_{n}(z) I_{n}^{\prime}(z) / K_{n}^{\prime}(z) I_{n}^{\prime}(z)-K_{n}^{\prime}(z) I_{n}^{\prime}(z)\right)$, qui apparaissent dans les solutions analytiques. Ces limitations sont surmontées ici grâce à l'utilisation des algorithmes de Brancik [31] dont l'implémentation dans MATLAB est immédiate et particulièrement efficace sur le plan numérique. La méthode semi-analytique ainsi proposée nous a permis de traiter le cas des coques élastiques couplées par le fluide, qui n'avait pas été résolu avec une approche similaire par Iakovlev.

\section{Exemple d'application}

Une application de la méthode de calcul est réalisée pour un problème caractérisé par les grandeurs suivantes $R_{\mathrm{i}}=0,5 \mathrm{~m}, R_{\mathrm{e}}=1 \mathrm{~m}, h_{\mathrm{e}}=h_{\mathrm{i}}=0,01, \rho_{S, \mathrm{i}}=\rho_{S, \mathrm{e}}=$ $7800 \mathrm{~kg} \cdot \mathrm{m}^{-3}, E_{\mathrm{e}}=E_{\mathrm{i}}=1,95 \times 10^{11} \mathrm{~Pa}, \rho_{\mathrm{F}, \mathrm{i}}=\rho_{\mathrm{F}, \mathrm{e}}=$ $1000 \mathrm{~kg} \cdot \mathrm{m}^{-3}, c_{\mathrm{i}}=c_{\mathrm{e}}=1400 \mathrm{~m} \cdot \mathrm{s}^{-1}$. Les paramètres de l'explosif sont $C=0,84 \mathrm{~kg}, D=5 \mathrm{~m}, a=0,33, b=1,13$ et $c=-0,22$.

La figure 5 montre le champ de pression obtenu à différents instants réduits par simulation numérique avec une décomposition de Fourier utilisant $N=120$ modes ortho-radiaux et permet ainsi de visualiser la réflexion et la radiation dans le fluide externe de l'onde incidente par la coque extérieure, la transmission de l'onde de choc dans le fluide interne et les réflexions/rayonnements multiples de l'onde de pression dans le fluide interne par les coques intérieure et extérieure.

La figure 6 donne l'évolution du champ de déplacement et de vitesse dans la direction radiale des coques intérieure et extérieure au droit de l'impact de l'onde de choc (i.e. en $\theta=0$ ) au cours du temps dans les premiers instants de l'interaction. Le profil de mise en vitesse des coques fait apparaître aux premiers instants une mise en vitesse « initiale » due au chargement de l'onde incidente sur les coques (de façon directe sur la coque intérieure, et par transmission de l'onde de choc au fluide - par effet de «transparence acoustique »- sur la coque intérieure). 


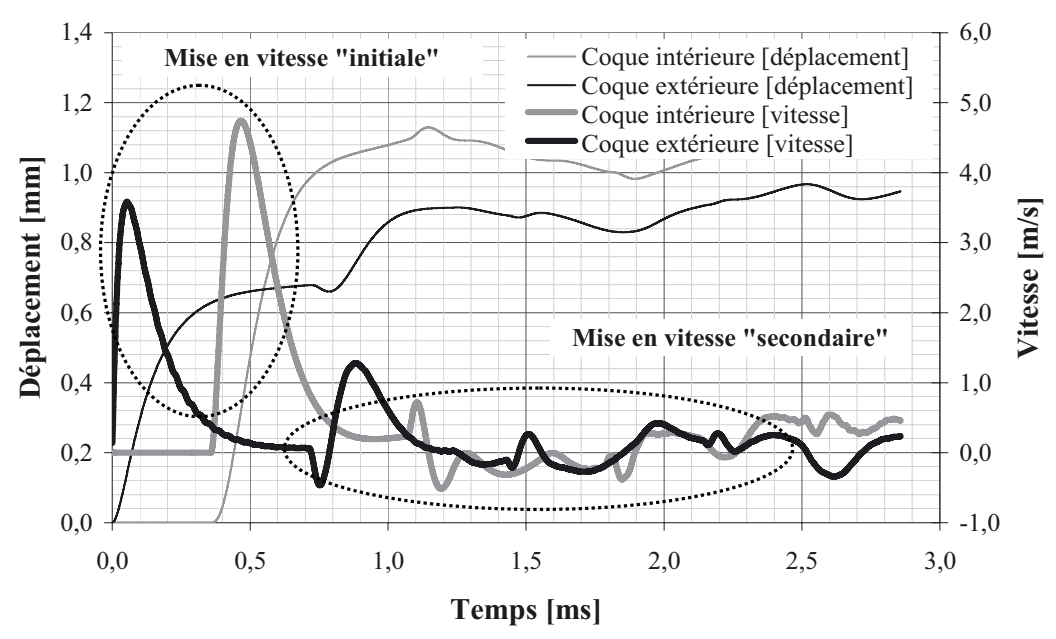

Fig. 6. Déplacement et vitesse de la coque extérieure et intérieure dans la direction radiale en $\theta=0$ en fonction du temps.

Ce profil de mise en vitesse est ainsi très similaire à celui obtenu en considérant la coque immergée seule. Dans les instants ultérieurs, on note l'existence d'une mise en vitesse « secondaire » conséquente du chargement en pression dans le fluide interne, par propagation et réflexions d'ondes dont la dynamique est régie par le couplage des mouvements des deux coques par le fluide contenu dans l'espace annulaire. Les simulations montrent ainsi que dans le cas étudié, les effets de couplage des coques par le fluide interne peuvent être négligés : le rapport des rayons des coques est ici tel que les effets de chargement « secondaire » de la coque extérieure par l'onde réfléchie et rayonnée dans le fluide interne par la coque intérieure n'interagissent pas avec le chargement « primaire » sur la coque extérieure dû à l'onde incidente dans le fluide externe.

Sur la figure 5, on note que l'onde réfléchie/rayonnée interne impacte l'intérieur de la coque extérieure au temps $t=0,71 \mathrm{~ms}$ (Fig. 5c), ce qui se traduit par un pic local sur la mise en vitesse de la coque extérieure en $\theta=0$ (voir Fig. 6). Sur la coque interne, un effet de rechargement par le fluide externe se produit au temps $t=1,06 \mathrm{~ms}$ (voir Fig. 6), au moment où l'onde acoustique qui se propage dans le fluide interne impacte la coque interne (voir Fig. 5d); à cet instant, les effets de chargement «primaire » dus à l'onde de choc incidente sont nettement atténués (voir Fig. 6). Pour ce système, les interactions entre les effets de l'onde incidente (dans les fluides interne et externe) et les ondes réfléchies/rayonnées par les coques dans le fluide confiné sont ainsi négligeables. Le cas étudié sommairement ici est cependant assez éloigné des géométries industrielles pour lesquelles les rapports des rayons des deux coques sont plus faibles : on peut s'attendre dans ce cas à ce que les effets de combinaison de l'onde incidente et des ondes réfléchies/rayonnées dans le fluide présent dans l'espace annulaire soient plus marqués. Ce point fera l'objet d'études plus détaillées qui permettront de quantifier l'influence des ratios géométriques (rapport des rayons intérieur/extérieur, rapport d'épaisseur/rayon, etc.) sur la réponse du système.

\section{Conclusion}

On a présenté dans cet article une méthode de calcul semi-analytique pour la simulation de l'interaction entre deux coques élastiques immergées dans (et couplées par) un fluide compressible et une onde de choc acoustique représentant le chargement correspondant à l'onde primaire consécutive à une explosion sous-marine.

En adoptant une modélisation linéaire du comportement des structures et des fluides en géométrie bidimensionnelle, il est possible de proposer une solution analytique au problème d'interaction fluide/structure ainsi posé. La résolution nécessite une décomposition analytique sur les modes de Fourier des champs de déplacement structure et de pression fluide et une opération numérique de transformation de Laplace.

Cette méthode de calcul proposée est développée dans un code de calcul dédié dans l'environnement MAT$\mathrm{LAB}$ et offre des perspectives complémentaires aux calculs qui peuvent être réalisés avec des codes industriels pour des problèmes aux géométries beaucoup plus complexes. Elle fournit en particulier des résultats multiphysiques exacts (au sens où les approximations introduites dans la modélisation sont maîtrisées) dans des géométries simples. Ces résultats peuvent par conséquent servir de cas de validation pour les codes industriels ou pour des études de pré-dimensionnement de structures navales.

\section{Références}

[1] R.H. Cole, Underwater explosions, Princeton University Press, 1948

[2] J.H. Haywood, Response of elastic cylindrical shell to pressure pulse, J. Mech. Appl. Math. 11 (1958) 126-141

[3] S.C. Tang, D.H.Y. Yen, Interaction of a plane acoustic wave with an elastic spherical shell, J. Acoustical Soc. Amer. 47 (1970) 1325-1233

[4] H. Huang, Transient interaction of plane acoustic wave with a spherical elastic shell, J. Acoustical Soc. Amer. 45 (1979) 661-670 
[5] T.L. Geers, C.L. Yen, Inelastic response of an infinite cylindrical shell to transient acoustic waves, J. Appl. Mech. 56 (1989) 900-909

[6] Y.W. Kwon, P.K. Fox, Underwater shock response of a cylinder subjected to side-on explosion, Computers \& Structures 48 (1993) 637-646

[7] Y.S. Shin, D.T. Hooker, Damage response of submerged imperfect cylindrical structures to underwater explosion, Computers \& Structures 60 (1995) 683-693

[8] C.C. Liang, C.Y. Hsu, W.H. Lai, A Study of transient response of a submerged spherical shell under shock waves, Ocean Eng. 28 (2000) 71-94

[9] A.H. Keil, The Response of ships to underwater explosions, Trans. Soc. Naval Architects Marine Eng. 69 (1961) 33-410

[10] I.K. Park, J.C. Kim, C.W. An, D.S. Cho, Measurement of naval ship response to underwater explosion shock loading, Shock Vibration 10 (2003) 365-377

[11] J.A. DeRuntz, The underwater shock analysis code and its applications, 60th Shock and Vibration Symposium, Virginia Beach, 14-16 November 1989

[12] H.U. Mair, Review: hydrocodes for structural response to underwater explosion. Shock Vibration 6 (1999) 81-96

[13] Y.W. Kwon, R.E. Cunningham, Comparison of USADYNA finite element models for a stiffened shell subjected to underwater shock, Computers \& Structures 66 (1998) 127-144

[14] H. Huang, Y.F. Wang, Early-times interaction of a spherical acoustic waves and cylindrical elastic shell, J. Acoustical Soc. Amer. 50 (1971) 885-891

[15] H. Huang, Y.F. Wang, Asymptotic fluid-structure interaction theories for acoustic radiation prediction, J. Acoustical Soc. Amer. 77 (1985) 1389-1394

[16] T.L. Geers, Residual potential and approximation methods for three-dimensional fluid-structure interaction problems, J. Acoustical Soc. Amer. 49 (1971) 1505-1510

[17] T.L. Geers, C.A. Felippa, Doubly asymptotic approximations for vibration analysis of submerged structures, J. Acoustical Soc. Amer. 73 (1983) 1152-1159

[18] G. Chertock, The Transient flexural vibrations of shiplike structures exposed to underwater explosions, J. Acoustical Soc. Amer. 48 (1970) 170-180
[19] Y.S. Shin, J.E. Chisum, Modeling and simulation of underwater shock problems using a coupled LagrangianEulerian analysis approach, Shock Vibration 4 (1997) 110

[20] Y.S. Shin, L.D. Santiago, Surface ship shock modeling and simulation: two-dimensional analysis, Shock Vibration 5 (1998) 129-137

[21] Y. Le Bras, D. Vienne, Underwater explosion response of an SSK, UDT Conference, Amsterdam, 22 June 2005

[22] C.C. Liang, Y.S. Tai, Shock response of surface ship subjected to noncontact underwater explosions, Ocean Eng. 33 (2006) 748-772

[23] F. Besnier, Simulation numérique et conception des structures de grands navires, Mécanique \& Industries 7 (2006) 213-222

[24] S. Iakovlev, External shock loading on a submerged fluidfilled cylindrical shell, J. Fluids Structures 22 (2006) 997-1028

[25] S. Iakovlev, Submerged fluid-filled cylindrical shell subjected to a shock wave: fluid-structure interaction effects, J. Fluids Structures 23 (2007) 117-142

[26] A. Pearson, J.R. Blake, A.R. Otto, Jets in bubble, J. Eng. Math. 48 (2004) 391-412

[27] D. Drikakis, D. Ofengeim, E. Timofeev, P. Voionovich, Computation of non stationary shock wave/cylinder interaction using adaptive grid methods, J. Fluids Structures 11 (1997) 665-691

[28] M.A. Sprague, T.L. Geers, A spectral element/finite element analysis of a ship like structure subjected to an underwater explosion, Computer methods in applied, Mechanics Eng. 195 (2006) 2149-2167

[29] F. Axisa, Modélisation des systèmes mécaniques, Hermès, 2001

[30] C. Leblond, Modélisation de phénomènes fortement instationnaires en milieu couplé, Application au dimensionnement de structures immergées aux explosions sousmarines, Thèse de doctorat, Université de Nantes, 6 décembre 2007

[31] L. Brancik, Utilization of Matlab in simulation of linear hybrid circuits, Radioengineering 12 (2003) 6-11 